\title{
Relationships among conspiratorial beliefs, conservatism and climate scepticism across nations
}

\author{
Matthew J. Hornsey ${ }^{1 \star}$, Emily A. Harris ${ }^{1}{ }^{1}$ and Kelly S. Fielding ${ }^{2}$ \\ Studies showing that scepticism about anthropogenic climate change is shaped, in part, by conspiratorial and conservative \\ ideologies are based on data primarily collected in the United States. Thus, it may be that the ideological nature of climate \\ change beliefs reflects something distinctive about the United States rather than being an international phenomenon. Here \\ we find that positive correlations between climate scepticism and indices of ideology were stronger and more consistent in the \\ United States than in the other $\mathbf{2 4}$ nations tested. This suggests that there is a political culture in the United States that offers \\ particularly strong encouragement for citizens to appraise climate science through the lens of their worldviews. Furthermore, \\ the weak relationships between ideology and climate scepticism in the majority of nations suggest that there is little inherent to \\ conspiratorial ideation or conservative ideologies that predisposes people to reject climate science, a finding that has encour- \\ aging implications for climate mitigation efforts globally.
}

\begin{abstract}
t has long been established that people's views on the reality of anthropogenic climate change are shaped, in part, by worldviews and ideologies ${ }^{1-10}$. For example, the theory of cultural cognition $^{11}$ argues that appraisals of scientific evidence can be shaped by the extent to which people endorse individualism (as opposed to communitarianism) and hierarchical values (as opposed to egalitarianism). People who subscribe to individualistic and hierarchical values are more likely than others to value elites, free enterprise and the status quo: as such, they are inclined to favour 'big business' and to view 'big government' with moral and ideological suspicion.

Given that many solutions to climate change involve government regulations on high-emission industries, it seems reasonable to argue that people high in individualism and low in egalitarianism will be relatively motivated to reject the science that humans are causing climate change. A recent meta-analysis supports this notion: endorsement of individualistic and hierarchical ideologies was more strongly associated with belief in climate change than were education, scientific literacy, activist/green identity and personal experience of extreme weather events ${ }^{4}$. The same metaanalysis revealed a tendency for people who locate themselves as relatively conservative on measures of political ideology to be more likely to express scepticism about anthropogenic climate change.
\end{abstract}

Another ideology that has been implicated in climate scepticism is conspiratorial ideation, defined as an underlying worldview or predisposition toward viewing events and circumstances as the product of conspiracies ${ }^{12,13}$. There are a number of conspiracy beliefs about climate science, most prominent of which is that it is a hoax perpetrated by scientists who see it as an opportunity to wield influence, secure funding or act out a green/Marxist agenda ${ }^{13-15}$. For some, however, individual conspiracy beliefs emerge from a unitary 'conspiracist' worldview: the notion that it is common practice for large networks of people with malevolent agendas to perpetrate mass hoaxes on the public in near-perfect secrecy ${ }^{12,16}$. For people who feel this is the way the world works, any conspiracy theory carries with it an intimation of plausibility and coherence, which helps explain positive correlations between diverse conspiracy theories, even mutually inconsistent ones ${ }^{12,17}$. In light of the conspiracy theories that exist in relation to climate change, it makes sense that people who are prone to conspiratorial ideation are predisposed to believe that evidence for anthropogenic climate change is exaggerated or manufactured. Indeed, at least two studies have shown that people's general willingness to endorse conspiracies is positively correlated with a range of attitudes that lie outside the scientific consensus, including climate scepticism ${ }^{1,2}$. Furthermore, exposure to conspiracy theories reduces pro-environmental intentions and increases climate scepticism ${ }^{18,19}$.

History tells us, however, that it is not inevitable that there will be broad-based ideological resistance to scientific consensus, even on issues that imply the need for government to regulate corporate interests. An example is the case of chlorofluorocarbons (CFCs). In 1976, the National Academy of Sciences released a report concluding that CFCs were contributing to depletion of ozone in the atmosphere $^{20}$. The evidence at the time was subject to uncertainties, yet by 1978 the United States had already put measures into place to ban its use in aerosols ${ }^{21}$. In 1985, scientists provided firmer evidence that CFCs were contributing to the ozone hole over the Antarctic ${ }^{22}$, and the regulatory response was immediate: the next year an international treaty was signed to phase out CFCs globally, and the treaty entered into force 15 months later ${ }^{21}$.

The parallels between the CFC-ozone link and the $\mathrm{CO}_{2}-\mathrm{cli}$ mate change link are revealing: in both cases, a scientific consensus emerged about an impending environmental crisis, the solution for which implied government regulation over commercial enterprises. Theoretically, then, both issues should have been subject to the same ideological objections from those who have commitments to individualism, or those who see official government pronouncements through the lens of conspiracy. Yet in the case of CFCs, there was little evidence of a grassroots, community-driven 'ozonesceptic' movement. There were indeed attempts from the chemical industry and from some members of government and the scientific

'School of Psychology, University of Queensland, Brisbane, Queensland, Australia. ${ }^{2}$ School of Communication \& Arts, University of Queensland, Brisbane, Queensland, Australia. *e-mail: m.hornsey@uq.edu.au 
a
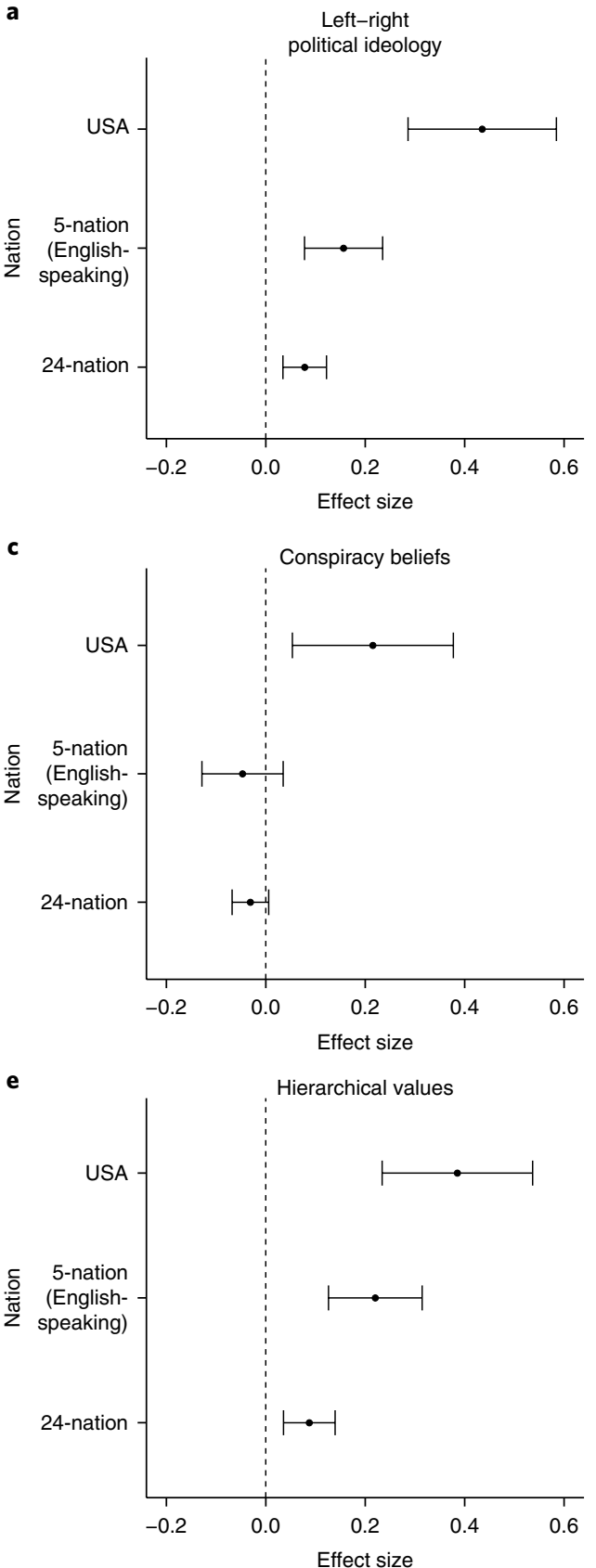

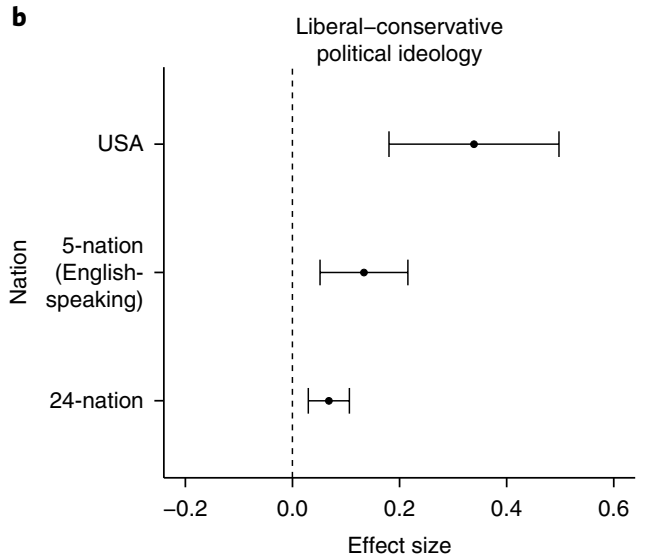

d

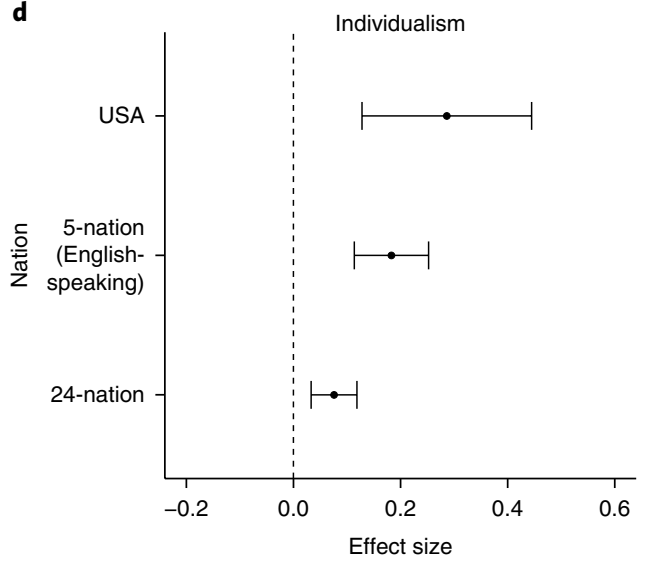

Fig. 1 | Relationships between climate change scepticism and ideological variables. a-e, Effect sizes for the relationship between climate change scepticism and left-right political ideology (a), liberal-conservative political ideology (b), conspiracy beliefs (c), individualism (d) and hierarchical values (e) for the United States compared to all other nations in the sample, and the five other English-speaking nations in the sample. Effect sizes for the United States are standardized regression coefficients. Effect sizes for 5- and 24-nation samples are pooled (averaged) correlation coefficients. The error bars show $95 \%$ confidence intervals.

community to cast doubt on the need for action ${ }^{23,24}$, but the actors were isolated and ultimately overwhelmed by bipartisan political action and community support. The swift and decisive response from government faced little organized resistance: as an example of environmental regulation based on science, the case of CFCs was a success story ${ }^{23}$.

One possible reason for this is that the stakes were relatively low: alternatives to Freon were in development, and transitioning required relatively little cost economically and societally. As such, one could argue that the corporate vested interests were not sufficiently threatened to warrant a serious campaign of misinformation, and so conservatives simply took the scientific consensus (and the regulatory implications of that consensus) on face value. This suggests that ideological barriers to accepting science do not just emerge 'bottom-up', in the sense of individuals spontaneously critiquing scientific consensus through the lens of their worldviews. Rather, ideological barriers to accepting science can also be cultivated and nourished 'top-down' by influential individuals and organizations who have a vested interest in communicating the notion that the science is flawed or inconclusive ${ }^{25}$. From this perspective, it can be argued that people with conservative and/or conspiratorial ideologies might not have focused their concerns on climate science if it were not for the fact that they had been coached to do so as part of a wider campaign. 


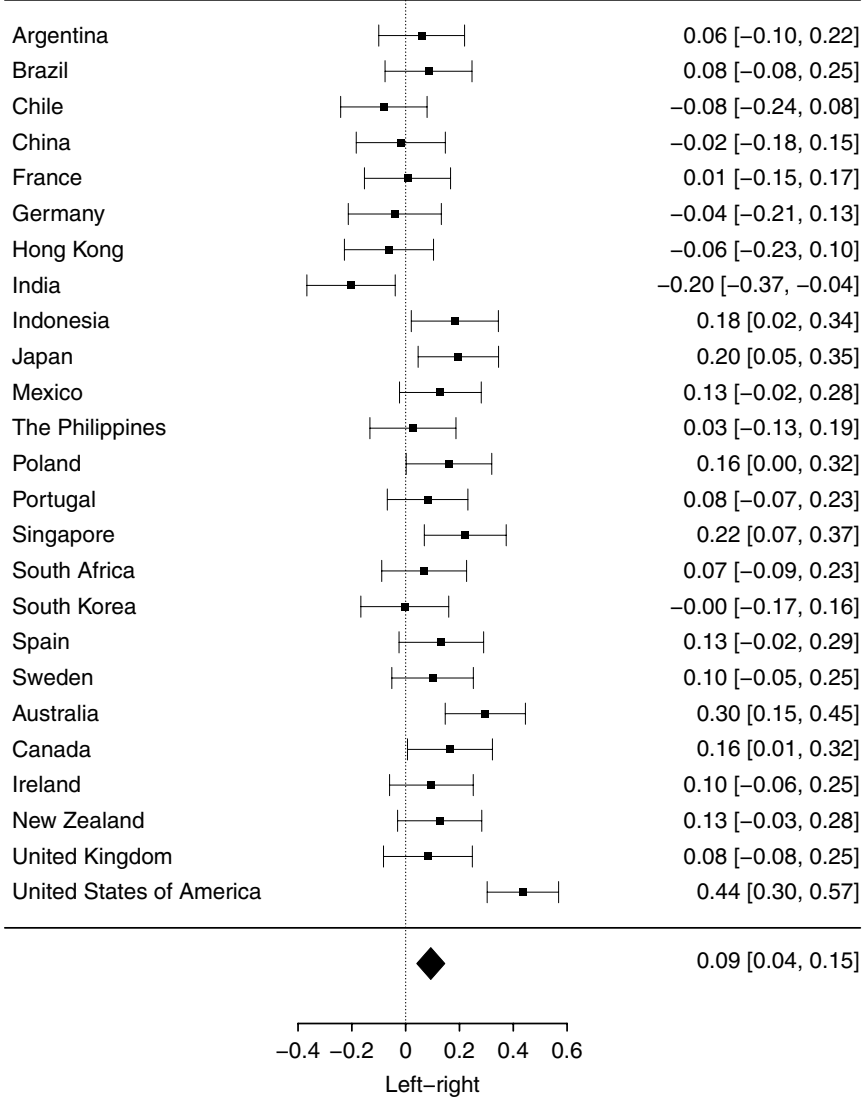

Fig. 2 | Relationship between left-right political ideology and climate change scepticism. Correlations between climate change scepticism and left-right political ideology across 25 samples. Western, Englishspeaking countries are grouped at the bottom. Tests of the difference between independent correlations using the Fisher $r$-to- $z$ transformation demonstrated the United States to have a significantly larger correlation than all other nations $(P<0.05)$ except Australia $(P=0.171)$. The error bars show $95 \%$ confidence intervals.

Oreskes and Conway ${ }^{23}$ identify how conservative think tanks have run campaigns of misinformation about the link between anthropogenic carbon emissions and climate change. As part of this campaign, disproportionate funding and mainstream media coverage have been given to scientists who are climate sceptics, many of whom have held long-standing ideological objections to government interference in the freedoms of individuals and industry. The case has been made that a network of vested interests, think tanks and ideologically driven scientists has succeeded in permeating the conservative political culture, locating climate science within the same culture wars that have traditionally divided people on issues such as gun control and abortion ${ }^{26-28}$.

One notable aspect of the case made by Oreskes and Conway ${ }^{23}$ is that the protagonists of their historical account-the politicians, think tanks and scientists that they argue have worked together to sow seeds of doubt about climate science-are situated predominantly in the United States. Political developments since the publication of the book in 2010 underscore just how much the United States has come to exemplify the political polarization associated with climate change. For example, of 17 candidates who campaigned to be the Republican nominee for the 2016 Presidential campaign, only a minority are on record as endorsing the scientific consensus around anthropogenic climate change ${ }^{29}$. As such, one could argue that it is only in the United States that climate scepticism has insinuated itself so successfully within the elite of a mainstream political

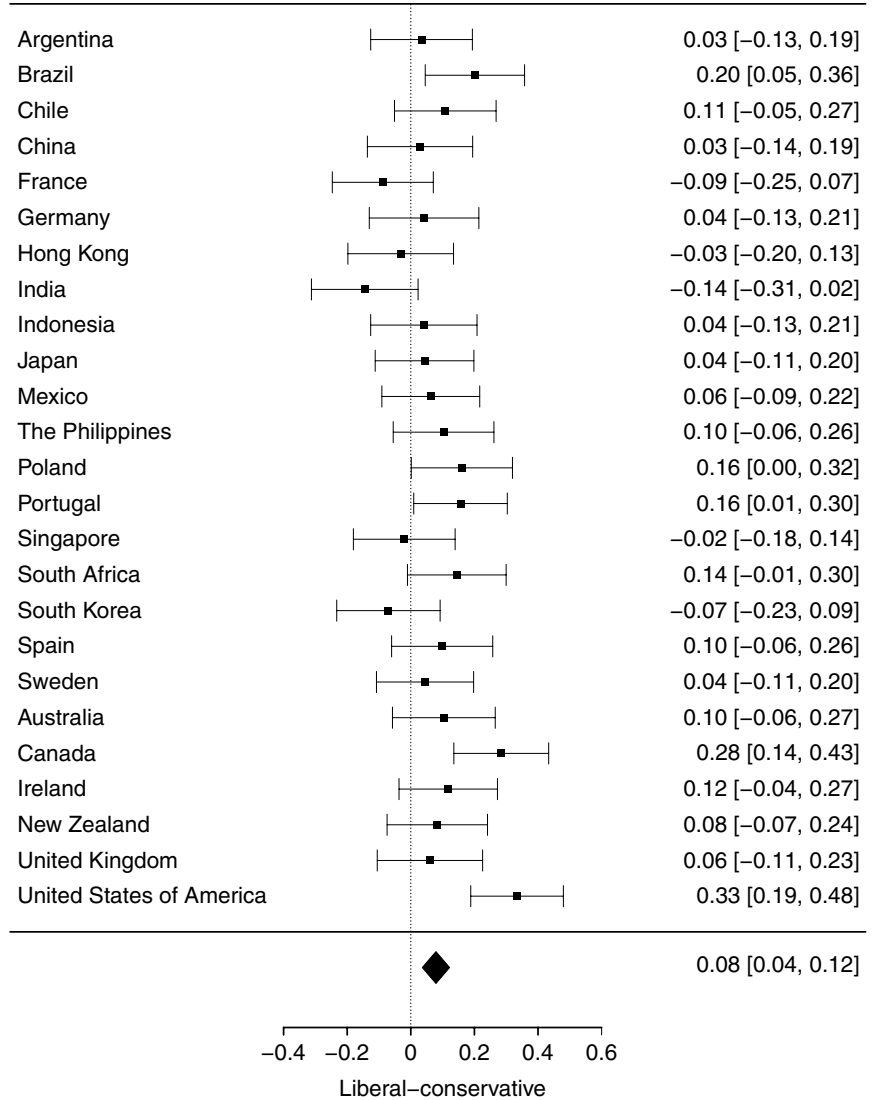

Fig. 3 | Relationship between liberal-conservative political ideology and climate change scepticism. Correlations between climate change scepticism and liberal-conservative political ideology across 25 samples. Western, English-speaking countries are grouped at the bottom. Tests of the difference between independent correlations using the Fisher $r$-to- $z$ transformation demonstrated the United States to have a significantly larger correlation than 19 of the nations $(P<0.05)$; a marginally larger correlation than Portugal $(P=0.097)$ and South Africa $(P=0.085)$; and no significant difference from Brazil $(P=0.230)$, Canada $(P=0.638)$ and Poland $(P=0.119)$. The error bars show $95 \%$ confidence intervals.

party, and that it has formed such a high-profile role within the political rhetoric.

This raises the question: if the United States is the front line in the ideological struggle to convince people of the reality or otherwise of anthropogenic climate change, could it also be the case that ideology will have a disproportionately large impact on Americans' belief in climate change? Another way of asking the same question is: is the role of ideology in predicting climate scepticism a genuinely international phenomenon, or is it most pronounced in the United States?

As the majority of research on the psychology of climate change is conducted in the United States, it is difficult to answer this question on the basis of existing research. Here we find that relationships between climate scepticism and measures of conspiratorial thinking and conservative ideology are larger in the United States compared to the pooled average of the other 24 nations tested, and the pooled average of the other 5 Western, English-speaking nations tested. Moreover, the United States is the only nation for which these correlations are significant across all five indices of ideology. In contrast, in approximately three-quarters of the cases, there is no significant relationship between the various indices of ideology and climate scepticism. The size of the relationship between climate scepticism and conservative ideologies is positively correlated with national 


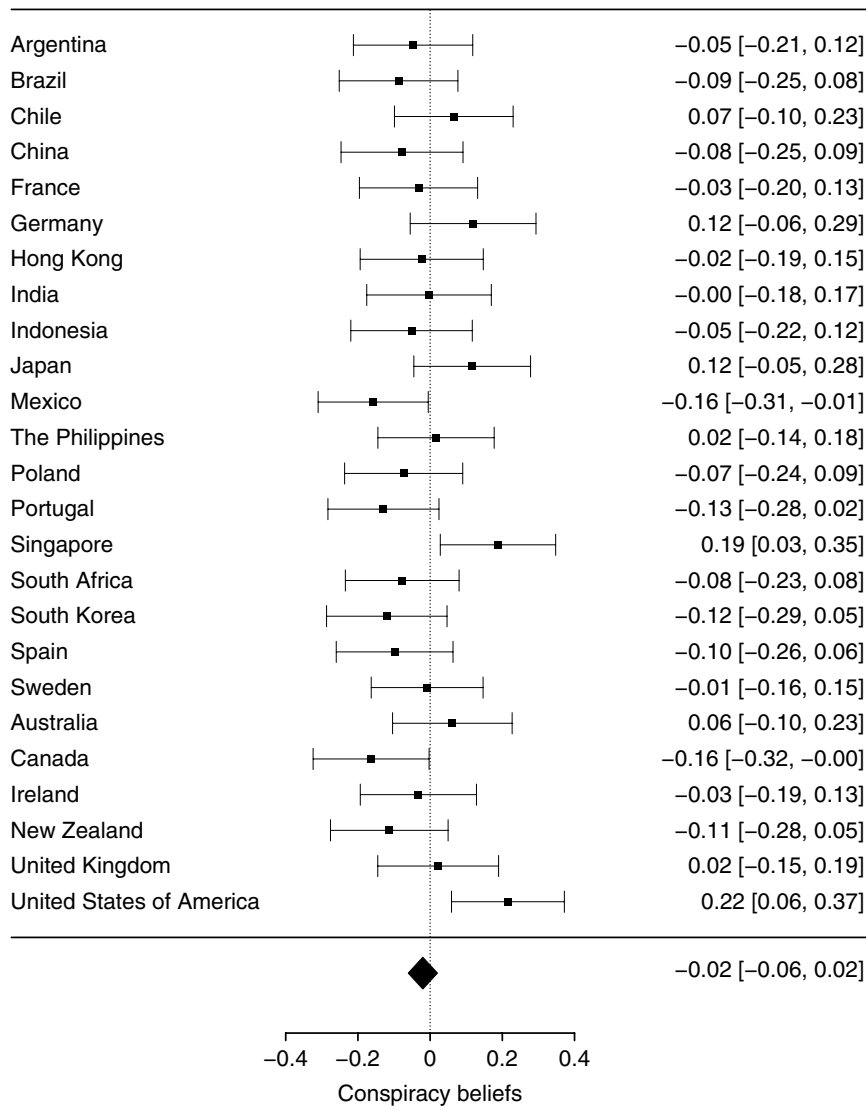

Fig. 4 | Relationship between conspiratorial beliefs and climate change scepticism. Correlations between climate change scepticism and conspiracy beliefs across 25 samples. Western, English-speaking countries are grouped at the bottom. Tests of the difference between independent correlations using the Fisher $r$-to-z transformation demonstrated the United States to have a significantly larger correlation than 15 of the nations $(P<0.05)$; a marginally larger correlation than India $(P=0.069)$, the Philippines $(P=0.085)$ and Sweden $(P=0.050)$; and no significant difference from Australia $(P=0.190)$, Chile $(P=0.200)$, Germany $(P=0.418)$, Japan $(P=0.390)$, Singapore $(P=0.810)$ and the United Kingdom $(P=0.101)$. The error bars show $95 \%$ confidence intervals.

per capita carbon emissions, an index that can be interpreted as a proxy for fossil fuel reliance within that nation.

Effects in the United States versus pooled-average effects We collected data from 5,323 participants from 25 nations/provinces. The samples approximated representativeness of the global population in terms of age, sex and political ideology (see Methods). We measured people's endorsement of individualist and hierarchical ideologies, as well as their belief in four internationally recognized conspiracy theories (surrounding the assassination of John F. Kennedy; the death of Princess Diana; the 9/11 terrorist attacks; and the existence of a New World Order). We also measured the extent to which people located themselves as politically right (versus left) and conservative (versus liberal). As these two indices of political ideology are often uncorrelated (or even negatively correlated) in some communist or ex-communist nations, we analysed them separately. Means and standard deviations within each of the 25 samples are summarized in Supplementary Table 1, and intercorrelations among the measures are summarized in Supplementary Table 2.

Figure 1 illustrates the correlation between our 5 measures of ideology and climate scepticism within the United States, and compares the size of that correlation with the average correlation of the other

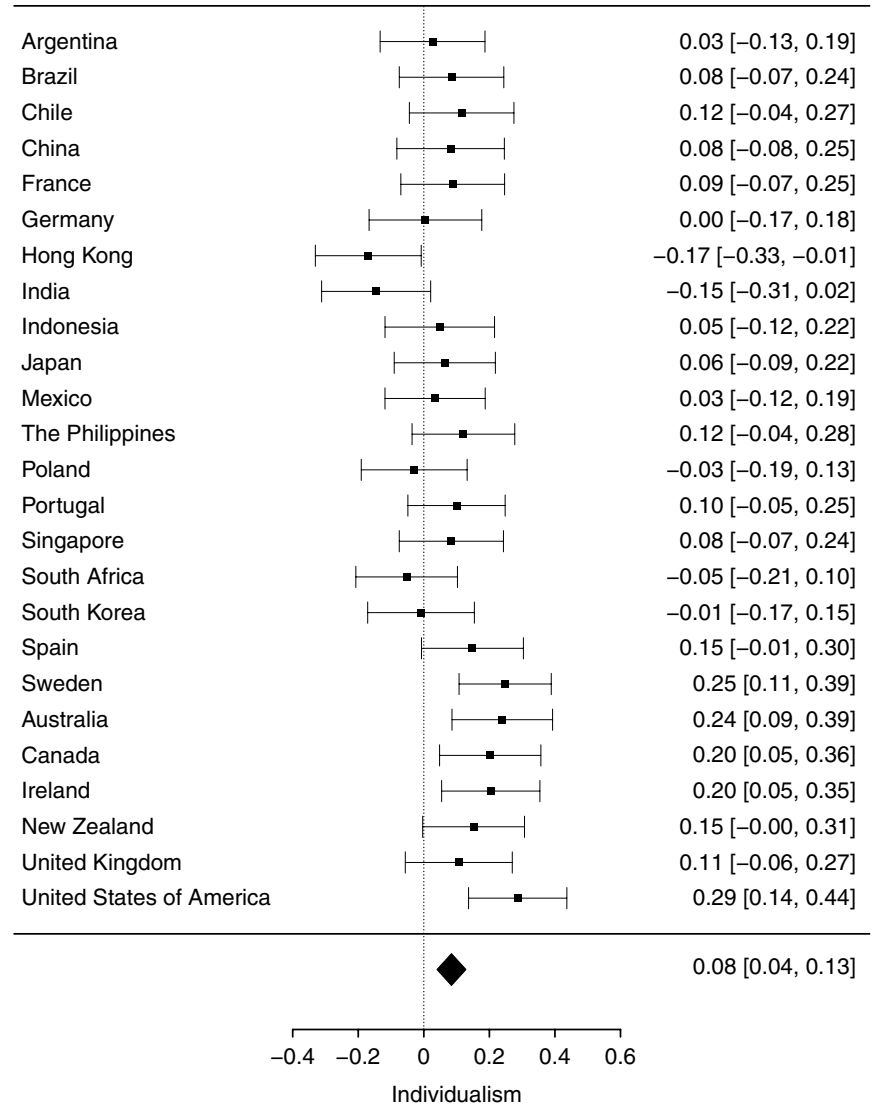

Fig. 5 | Relationship between individualism and climate change scepticism. Correlations between climate change scepticism and individualism across 25 samples. Western, English-speaking countries are grouped at the bottom. Tests of the difference between independent correlations using the Fisher $r$-to- $z$ transformation demonstrated the United States to have a significantly larger correlation than ten of the nations $(P<0.05)$; a marginally larger correlation than Brazil $(P=0.075)$, China $(P=0.075)$, France $(P=0.078)$, Portugal $(P=0.089)$ and Singapore $(P=0.074)$; and no significant difference from Australia $(P=0.667)$, Canada $(P=0.453)$, Chile $(P=0.131)$, Ireland $(P=0.453)$, New Zealand $(P=0.230)$, the Philippines $(P=0.142)$, Spain $(P=0.215)$, Sweden $(P=0.719)$ and the United Kingdom $(P=0.116)$. The error bars show $95 \%$ confidence intervals.

24 nations combined (calculated using the $\mathrm{R}$ metafor package ${ }^{30}$ ). To formally compare the relationship between ideology and climate scepticism for the United States as compared to the other 24 nations combined, we calculated effect sizes for the United States using linear regression models. We then used the lme4 package in $\mathrm{R}^{31}$ to calculate effect sizes across the other 24 nations, using mixed-effects models that accounted for the nesting of people (Level 1) within countries (Level 2). Using unstandardized $b$ values and standard errors for each relationship between ideology and climate change scepticism, we calculated $z$ scores to test for differences in the size of the effects between the United States and the other 24 nations. On all 5 measures, the size of the relationships in the US sample was significantly stronger than the size of the relationship in the pooled 24 -nation sample (all $z$ values $>2.32$, all $P$ values $<0.021$ ). Note that the data reported here are based on analyses conducted after taking out participants who failed the attention check (see Methods). However, all of the effects were still significant regardless of whether the attention check fails were included or excluded. Furthermore, all effects remained significant when age, sex and education differences were controlled for (see Supplementary Table 3). 


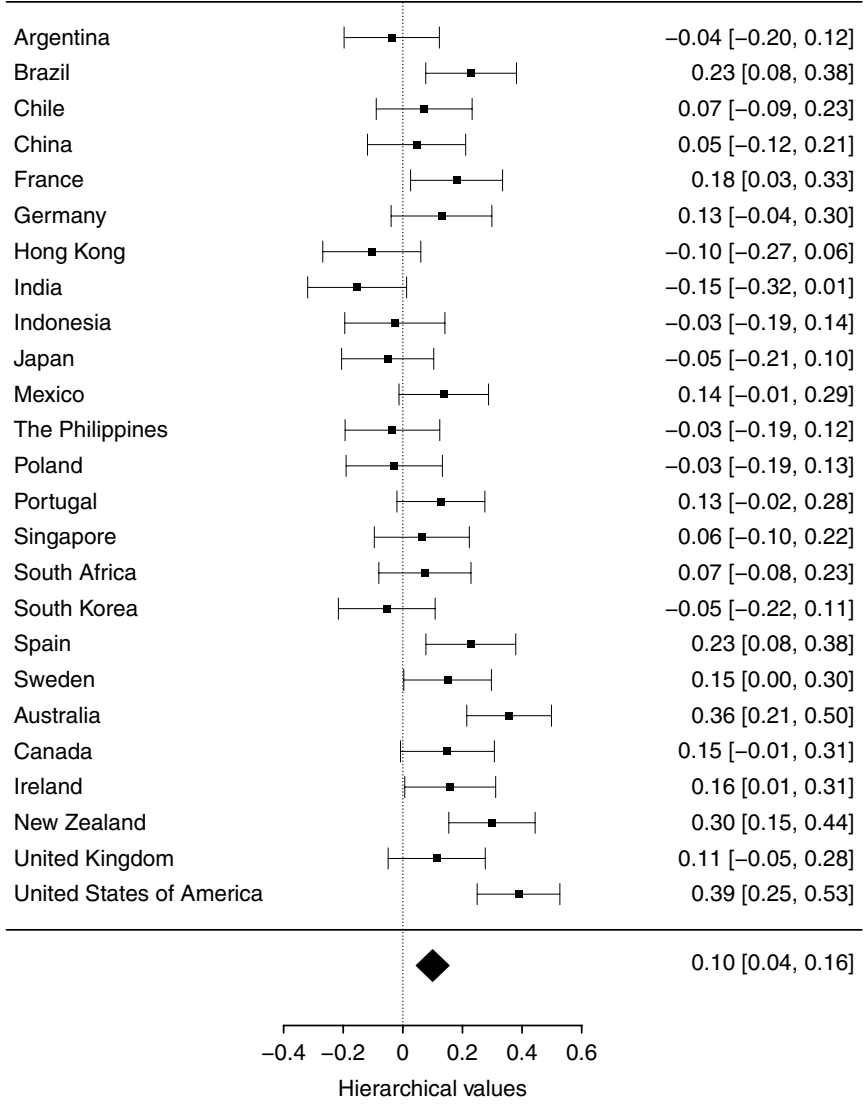

Fig. 6 | Relationship between hierarchical values and climate change scepticism. Correlations between climate change scepticism and hierarchical values across 25 samples. Western, English-speaking countries are grouped at the bottom. Tests of the difference between independent correlations using the Fisher $r$-to- $z$ transformation demonstrated the United States to have a significantly larger correlation than 19 of the nations $(P<0.05)$; a marginally larger correlation than France $(P=0.052)$; and no significant difference from Australia $(P=0.764)$, Brazil $(P=0.134)$, New Zealand $(P=0.390)$ and Spain $(P=0.131)$. The error bars show $95 \%$ confidence intervals.

Although we took care to ensure fidelity of the translations and to eliminate culturally specific wording in the questionnaires (see Methods), it is still possible that the translations of scales may have been imperfect, or that there was a Western-centric aspect to the scales that privilege our hypothesis. To provide a more conservative test of our prediction, we also compared the strength of the relationships within the United States and the pooled correlations among the five other English-speaking Western nations (using the same technique described above). For four of the five indices of ideology, the size of the relationships in the US sample was significantly larger than the size of the relationships in this five-nation sample (all $z$ values $>$ 1.98 , all $P$ values $<0.048)$. The exception was individualism, for which the comparison was not significantly different $(z=1.07, P=0.287)$. Again, the analyses reported here were conducted after excluding participants who failed the attention check; if the full data set is used then all the comparisons (including individualism) are significantly different $(z=2.10, P=0.036)$. Interpretation of the effects did not change when age, sex and education were controlled for.

\section{Effects in the United States compared with each nation}

Figures 2-6 show the relationships between climate scepticism and indices of ideology separately for each nation. Two patterns emerge from these data. First, for each of the 5 indices of ideology (conspiratorial ideation, individualism, hierarchy, left-right political ideology and liberal-conservative political ideology), the US sample had the largest correlation coefficients of the 25 samples. Furthermore, the United States is the only country for which the correlations were significant in all five indices (Australia was the next most consistent, with three out of five correlations significant). Thus, there is a consistent pattern suggesting that the relationship between ideology and climate scepticism is particularly strong in the United States.

Second, Figs. 2-6 show that, in approximately three-quarters of cases, there is no significant relationship between the various indices of ideology and scepticism. However, some nations do show significant correlations on some measures, and in several cases the correlations-while smaller than that observed in the United States-are not significantly smaller. As such, one could make the case that the United States is not so much an outlier as at the upper end of a distribution. For example, Brazil, Australia and Canada each display two or more significant relationships between our indices of ideology and climate scepticism. This is consistent with previous studies showing positive relationships between climate scepticism and various indices of conservative ideology in those countries ${ }^{32-34}$. It also dovetails with the observations of researchers in political science and media studies, who have written about conservative politicians and think tanks in Australia and Canada, and their role in deploying ignorance-building strategies to manage public impressions of climate change $\mathrm{e}^{35,36}$. In contrast, relationships between conservative ideologies and climate scepticism appear to be relatively weak and inconsistent in Europe and the United Kingdom. Previous research has shown significant positive relationships between indices of conservatism and climate scepticism in the United Kingdom ${ }^{37}$ and Western Europe ${ }^{38}$; however, the standardized coefficients in those papers are comparable in size to those reported here. Overall, the data suggest a trend such that conservative ideologies are correlated with climate scepticism in these regions, but the size of these effects is relatively modest.

As is typical when English-constructed scales are measured in other languages and cultures, the reliability of our scales did tend to be slightly stronger in the Western than in the non-Western contexts (see Supplementary Table 5). However, the alphas were respectable in the vast majority of cases ( $>90 \%$ exceeded the conventional 0.60 criterion for an acceptable alpha). Furthermore, the disattenuated correlations that correct for scale reliability were only subtly different from the original bivariate correlations (see Supplementary Table 6). Even after adjusting for reliabilities, the United States has the largest correlation for all five indices of ideology, and the United States is the only country for which the correlations were significant across all five indices.

\section{Per capita carbon emissions as an explanatory variable}

In reflecting on the variability across countries, it is notable that nations that displayed the largest relationships (for example, the United States, Australia, Canada and Brazil) tend to be those whose economies are relatively highly reliant on fossil fuel industries. To follow up this observation, we conducted meta-regression analyses to test whether per capita carbon emissions within a nation could help explain the variability in the strength of association between the ideological variables and climate scepticism across samples. These analyses revealed that greater national per capita carbon emissions was associated with stronger relationships between climate change scepticism and endorsement of hierarchical $(b=0.06$, s.e. $=0.03, P=0.012)$, right-wing $(b=0.08$, s.e. $=0.02, P<0.001)$, conservative $(b=0.04$, s.e. $=0.02, P=0.043)$ and individualist ideologies $(b=0.04$, s.e. $=0.02, P=0.037)$. A similar effect on conspiracy beliefs was non-significant $(b=0.03$, s.e. $=0.02, P=0.153)$. Although the causal mechanism for these effects cannot be demonstrated, it may be that per capita carbon emissions is a proxy for vested interests around climate change, 
both collectively (in terms of the fossil fuel industry's investment in that country) and individually (in terms of the perceived sacrifices and changes that citizens feel they need to make to live a low-carbon lifestyle). The greater the vested interests in resisting change, the more incentive there is to engage-and believe-in ideologically driven campaigns of misinformation about the reality of anthropogenic climate change.

\section{Limitations and conclusions}

A strength of our data is their ability to provide like-for-like comparisons for 25 countries across the globe. However, the data also carry limitations. For example, we used a single-item measure of climate scepticism, which focused entirely on beliefs about the causes of climate change. Future research should broaden this investigation to include other types of climate scepticism, for example, scepticism about the presumed rate of change, the impact of that change and the need for mitigation responses ${ }^{37,39}$. We also acknowledge that our data reflect cross-sectional correlations. As such, we are unable to disentangle the extent to which the ideologies are causing scepticism, scepticism is causing the ideologies or (as seems likely) they influence each other in a dynamic fashion.

Despite these limitations, the data suggest that in some countries political cultures have emerged that encourage citizens to appraise climate science through the lens of their conservative ideologies. Furthermore, the data demonstrate that the relationship between conservative ideologies and climate scepticism is unusually strong and consistent within the United States compared to other countries. For those who are embedded in US politics (and US-centric data sets), it is easy to see how one could reach the pessimistic conclusion that the debate around climate change is deeply mired in conspiratorial thinking and/or politically polarized 'culture wars'. However, a broader narrative emerging from our data is that there is nothing inherent to conspiratorial ideation or conservative ideologies that predisposes people to reject climate science. Indeed, for the majority of countries we sampled, one could not reliably predict participants' views on climate change from knowing whether they were chronically prone to conspiratorial thinking, or where they lay in terms of being individualist or communitarian, hierarchical or egalitarian, left or right, liberal or conservative. In these samples at least, one can be optimistic that climate science is more likely to be appraised on its merits, rather than being tainted by the desire to reach conclusions that are compatible with one's worldviews.

\section{Methods}

Methods, including statements of data availability and any associated accession codes and references, are available at https://doi. org/10.1038/s41558-018-0157-2.

Received: 5 December 2017; Accepted: 5 April 2018; Published online: 7 May 2018

\section{References}

1. Lewandowsky, S., Gignac, G., \& Oberauer, K. The role of conspiracist ideation and worldviews in predicting rejection of science. PloS One $\mathbf{8}$, e75637 (2013).

2. Lewandowsky, S., Oberauer, K. \& Gignac, G. NASA faked the Moon landing therefore (climate) science is a hoax: An anatomy of the motivated rejection of science. Psychol. Sci. 24, 622-633 (2013).

3. McCright, A. M. \& Dunlap, R. E. The politicization of climate change and polarization in the American public's views of global warming, 2001-2010. Sociol. Q. 52, 155-194 (2011).

4. Hornsey, M. J., Harris, E. A., Bain, P. G. \& Fielding, K. S. Meta-analyses of the determinants and outcomes of belief in climate change. Nat. Clim. Change 6, 622-626 (2016)

5. Kahan, D. M. et al. The polarizing impact of science literacy and numeracy on perceived climate change risks. Nat. Clim. Change 2, 732-735 (2012).
6. Bain, P. G., Hornsey, M. J., Bongiorno, R. \& Jeffries, C. Promoting proenvironmental action in climate change deniers. Nat. Clim. Change 2, 600-603 (2012).

7. Bliuc, A.-M. et al. Public division about climate change rooted in conflicting socio-political identities. Nat. Clim. Change 5, 226-229 (2015).

8. Hornsey, M. J. \& Fielding, K. S. Attitude roots and jiu jitsu persuasion: Understanding and overcoming the motivated rejection of science. Am. Psychol. 72, 459-473 (2017).

9. Kahan, D. M., Jenkins-Smith, H. \& Braman, D. Cultural cognition of scientific consensus. J. Risk Res. 14, 147-174 (2011).

10. Leiserowitz, A. Climate change risk perceptions and policy preferences: The role of affect, imagery, and values. Climatic Change 77, 45-72 (2006)

11. Kahan, D. M. Fixing the communications failure. Nature 463, 296-297 (2010).

12. Wood, M. J., Douglas, K. M. \& Sutton, R. M. Dead and alive: Beliefs in contradictory conspiracy theories. Social. Psychol. Personal. Sci. 3, 767-773 (2012).

13. Uscinski, J., Douglas, K. \& Lewandowsky, S. in The Oxford Encyclopedia of Climate Change Communication (ed. Nisbet, M.) http://doi.org/cnjt (Oxford Univ. Press, Oxford, 2017).

14. Douglas, K. \& Sutton, R. Climate change: Why the conspiracy theories are dangerous. Bull. Atom. Sci. 71, 98-106 (2015).

15. Goertzel, T. Conspiracy theories in science. EMBO Rep. 11, 493-499 (2010).

16. Goertzel, T. Belief in conspiracy theories. Political Psychol. 15, 731-742 (1994).

17. Lewandowsky, S., Cook, J. \& Lloyd, E. The 'Alice in Wonderland' mechanics of the rejection of (climate) science: Simulating coherence by conspiracism. Synthese 195, 175-196 (2018).

18. Jolley, D. \& Douglas, K. M. The social consequences of conspiracism: Exposure to conspiracy theories decreases intentions to engage in politics and to reduce one's carbon footprint. Br. J. Psychol. 105, 35-56 (2014).

19. Van Der Linden, S. The conspiracy-effect: Exposure to conspiracy theories (about global warming) decreases pro-social behaviour and science acceptance. Pers. Indiv. Differ. 87, 171-173 (2015).

20. Committee on Impacts of Stratospheric Change Halocarbons: Environmental Effects of Chlorofluoromethane Release (National Academy of Sciences, Washington DC, 1976).

21. Morrisette, P. M. The evolution of policy responses to stratospheric ozone depletion. Nat. Resour. J. 29, 793-820 (1989).

22. Farman, J. C., Gardiner, B. G. \& Shanklin, J. D. Large losses of total ozone in Antarctica reveal seasonal $\mathrm{CIO}_{x} / \mathrm{NO}_{x}$ interaction. Nature 315, 207-210 (1985).

23. Oreskes, N. \& Conway, E. M. Merchants of Doubt: How a Handful of Scientists Obscured the Truth on Issues from Tobacco Smoke to Global Warming (Bloomsbury, New York, 2010).

24. Dotto, L. \& Schiff, H. The Ozone War (Doubleday, New York, 1978).

25. Carmichael, J. T. \& Brulle, R. J. Elite cues, media coverage, and public concern: An integrated path analysis of public opinion on climate change, 2001-2013. Environ. Polit. 26, 232-252 (2017).

26. McCright, A. M. \& Dunlap, R. E. Anti-reflexivity: The American conservative movement's success in undermining climate science and policy. Theory Cult. Soc. 27, 100-133 (2010).

27. Lewandowsky, S., Oreskes, N., Risbey, J. S., Newell, B. R. \& Smithson, M. Seepage: Climate change denial and its effect on the scientific community. Glob. Environ. Change 33, 1-13 (2015).

28. Jacques, P. J., Dunlap, R. E. \& Freeman, M. The organisation of denial: Conservative think tanks and environmental scepticism. Environ. Polit. 17, 349-385 (2008).

29. Kaplan, R. \& Uchimiya, E. Where the 2016 Republican candidates stand on climate change. CBS News (1 September 2015); https://www.cbsnews.com/ news/where-the-2016-republican-candidates-stand-on-climate-change

30. Viechtbauer, W. Conducting meta-analyses in $\mathrm{R}$ with the metafor package. J. Stat. Softw. 36, 1-48 (2010).

31. Bates, D., Mächler, M., Bolker, B. \& Walker, S. Fitting linear mixed-effects models using lme4. J. Stat. Softw. 67, 1-48 (2015).

32. Cook, J. \& Lewandowsky, S. Rational irrationality: Modeling climate change belief polarization using Bayesian networks. Top. Cogn. Sci. 8, 160-179 (2016)

33. Jylhä, K. M., Cantal, C., Akrami, N. \& Milfont, T. L. Denial of anthropogenic climate change: Social dominance orientation helps explain the conservative male effect in Brazil and Sweden. Pers. Indiv. Differ. 98, 184-187 (2016).

34. Fielding, K. S., Head, B. W., Laffan, W., Western, W. \& Hoegh-Guldberg, O. Australian politicians' beliefs about climate change: Politicial partisanship and political ideology. Environ. Polit. 21, 712-733 (2012).

35. Young, N. \& Coutinho, A. Government, anti-reflexivity, and the construction of public ignorance about climate change: Australia and Canada compared. Glob. Environ. Polit. 13, 89-108 (2013). 
36. McKeown, E. Talking points ammo: The use of neoliberal think tank fantasy themes to deligitmise scientific knowledge of climate change in Australian newspapers. Journalism Stud. 13, 277-297 (2012).

37. Capstick, S. B. \& Pidgeon, N. F. What is climate change scepticism? Examination of the concept using a mixed methods study of the UK public. Glob. Environ. Change 24, 389-401 (2014).

38. McCright, A. M., Dunlap, R. E. \& Marquart-Pyatt, S. T. Political ideology and views about climate change in the European Union. Environ. Polit. 25, 338-358 (2016).

39. Poortinga, W., Spence, A., Whitmarsh, L., Capstick, S. \& Pidgeon, N. F. Uncertain climate: An investigation into public scepticism about anthropogenic climate change. Glob. Environ. Change 21, 1015-1024 (2011).

\section{Acknowledgements}

The work reported in the current paper was supported by funding from the Australian Research Council (DP120100961).

\section{Author contributions}

M.J.H. and K.S.F. developed the study concept and design. E.A.H. led the process of conducting translations and collecting the data. E.A.H. also led the analysing and writing up of the results. M.J.H. drafted the manuscript, and all authors contributed to revisions.

\section{Competing interests}

The authors declare no competing interests.

\section{Additional information}

Supplementary information is available for this paper at https://doi.org/10.1038/ s41558-018-0157-2.

Reprints and permissions information is available at www.nature.com/reprints. Correspondence and requests for materials should be addressed to M.J.H.

Publisher's note: Springer Nature remains neutral with regard to jurisdictional claims in published maps and institutional affiliations. 


\section{Methods}

Participants. Data were collected between 31 March and 11 May 2016, using the data collection company Survey Sampling International (SSI). We targeted respondents from 25 regions: Argentina, Australia, Brazil, Canada, Chile, China, France, Germany, Hong Kong, India, Indonesia, Ireland, Japan, Mexico, New Zealand, the Philippines, Poland, Portugal, Singapore, South Africa, South Korea, Spain, Sweden, United Kingdom and the United States. Before beginning the survey, potential participants were asked 'what country do you live in?' Respondents were screened out of the study if they stated that they lived in a nation other than the nation being sampled $(n=692)$. Participants with more than $90 \%$ data points missing (participant drop-outs) were also excluded from analyses $(n=60)$. This left 5,323 usable participants. For demographic information broken down by sample, see Supplementary Table 4 . Of the 5,323 respondents, 1,291 failed an attention check ('To show that you are reading the questions carefully, please select Slightly Disagree here') and so were excluded from the main analyses. Participants received monetary compensation for their time, which was determined by SSI and adjusted for each country, such that the purchasing power of each reimbursement was consistent across countries.

SSI was chosen due to their emphasis on representativeness of panel respondents, whereby their panel comprises relatively representative distributions of gender and age within nations. As can be seen in Supplementary Table 4, our overall sample was indeed balanced with respect to gender ( $49.9 \%$ female). Given that participants needed to be 18 or over to complete the survey, one would expect that our sample would be older than the population of the respective countries. Even so, the median ages of our samples were, on average, only 4.32 years older than the median age of the respective national populations. Furthermore, inspection of Supplementary Table 1 shows that the samples were relatively balanced in terms of political orientation: scores on the left-right dimension were slightly above the mid-point, whereas scores on the liberal-conservative dimension were slightly below the mid-point.

One limitation of online samples is that they tend to be more educated than the general population. Over-representation of tertiary educated people among the 16 Organisation for Economic Co-operation and Development (OECD) nations in our sample was relatively modest (on average, there was only a $4.01 \%$ difference between the percentage of tertiary educated participants in our sample and the percentage of tertiary educated people in the respective populations of the 16 OECD nations). For non-OECD nations, however, the over-representation of educated participants was more pronounced. As can be seen in Supplementary Table 2, however, age and education had very weak relationships with climate change scepticism; furthermore, controlling for these variables did not change the interpretation of the results.

Materials. Questionnaires were translated into the native language of non-English speaking samples using translation/back-translation procedures, and concerns about comprehension and translatability were flagged and addressed during that process. The first three predictors (conspiracy beliefs, individualism and hierarchy) were presented in a randomized order. These predictors were followed by a measure of the outcome variable (climate change scepticism) and finally by the measures of political ideology and demographics. Internal reliabilities for each nation across each of the scales are summarized in Supplementary Table 5. Supplementary Table 6 summarizes the intercorrelations between climate scepticism and each measure of ideology, reported both as bivariate correlations and as disattenuated correlations (controlling for scale reliability).

Conspiracy beliefs. Of the original 14 items used to measure conspiracy beliefs in an earlier study ${ }^{1}$, we used 4 . To avoid conflating conspiracy beliefs with a more specific 'mistrust in science' construct, we excluded any items that involved conspiracies about science (that is, items relating to severe acute respiratory syndrome, climate change, the moon landings and AIDS). We also excluded items that seemed to have more specific US impact rather than international impact (that is, items relating to Pearl Harbor, Martin Luther King, the Oklahama bombers and the formula of Coca-Cola). Of the remaining items, we also excluded two related to alien spacecraft (Rockwell and Area 51) that we felt might not resonate with a broad international audience. The remaining four items (endorsement of conspiracies surrounding the assassination of President John Kennedy, the death of Princess Diana, the existence of a New World Order and US government knowledge of the 9/11 terrorist attacks) fit our criteria of having broad international recognition and so were included. The 9/11 terrorist attacks are well-known internationally, and conspiracy theories that it represented an inside job' are popular throughout the non-Western world. The death of Princess Diana was also an internationally recognized incident, and conspiracy theories about this remain prevalent ${ }^{40}$. The other two conspiracy theories-about the New World Order and the death of John F. Kennedy-are among the most popular conspiracy theories in the world.

Participants rated their agreement with the conspiracy statement on a fivepoint scale $(1=$ strongly disagree; $5=$ strongly agree $)$ but with a sixth option for people who 'don't know'. Participants choosing the 'don't know' option were treated as missing values on this scale. Consistent with the notion of conspiracy beliefs forming part of a unitary worldview, endorsement of each conspiracy theory was positively correlated and so the four items were combined into a single scale $(\alpha=0.79)$. Note that the conspiratorial beliefs scores have been reported in another paper that focused on anti-vaccination attitudes, did not make reference to climate change and did not explore cross-national differences ${ }^{41}$.

Individualism-communitarianism worldview. Individualism-communitarianism was originally measured with 17 items from the Cultural Cognition Worldview $S_{\text {cale }}{ }^{42}$. Example items include 'The government interferes far too much in our everyday lives', 'Society works best when it lets individuals take responsibility for their own lives without telling them what to do', 'Government regulations are almost always a waste of everyone's time and money' and 'It's society's responsibility to make sure everyone's basic needs are met' (reversed). Items were responded to on a six-point scale ( $1=$ strongly disagree; $6=$ strongly agree). The scale showed modest internal reliability across the overall sample $(\alpha=0.71)$, but closer investigation revealed that the five negatively worded items coalesced poorly with each other $(\alpha=0.56)$. Consequently, we conducted our analyses just on the 12 positively worded items, which together showed relatively strong internal reliability $(\alpha=0.77)$.

Hierarchy-egalitarianism worldview. Hierarchy-egalitarianism was measured with 12 items from the Cultural Cognition Worldview Scale ${ }^{42}$. Example items include 'We have gone too far in pushing equal rights in this country' and 'A lot of problems in our society today come from the decline in the traditional family, where the man works and the woman stays home'. Two items were adapted to avoid cultural specificity. First, 'It seems like blacks, women, homosexuals and other groups don't want equal rights, they want special rights just for them' was adapted to read 'It seems like many minority groups in society don't want equal rights, they want special rights just for them'. Second, 'We need to dramatically reduce inequalities between the rich and the poor, whites and people of color, and men and women' was adapted to read 'We need to dramatically reduce inequalities between different groups of people in society, for example, the rich and the poor, and men and women'. A 13th item contained in the original scale-'Nowadays it seems like there is just as much discrimination against whites as there is against blacks'-was not included in the survey. All items were responded to on a six-point scale $(1=$ strongly disagree; $6=$ strongly agree). As for the scores on individualism, the scores on hierarchy showed modest internal reliability $(\alpha=0.66)$ that was improved when one just focused on the six positively worded items $(\alpha=0.69)$, and so analyses were conducted on only these items.

Climate change scepticism. Participants were asked 'Thinking about the causes of climate change, which of the following best describes your opinion?' This question was adapted from previous research ${ }^{39}$. The response options were: 'entirely caused by natural processes', 'mainly caused by natural processes', 'mainly caused by human activity', 'entirely caused by human activity', 'there is no such thing as climate change' and 'I don't know'. Of the overall sample, $21.2 \%$ maintained that climate change is 'entirely' or 'mainly' caused by natural processes. The most common response-comprising $72.4 \%$ of the sample-was to say that climate change is 'mainly' or 'entirely' caused by human activity. Only $1.5 \%$ of participants chose the option 'There is no such thing as climate change'. A small number of respondents (4.9\%) clicked an option saying 'I don't know', and were excluded from analyses.

We analysed the data by treating climate change scepticism as a continuous measure. People who said that climate change was 'entirely caused by human activity' were coded as 1 , 'mainly caused by human activity' as 2 , 'mainly caused by natural processes' as 3 , and 'entirely caused by natural processes' as 4 . As only a very small number of respondents clicked the option saying there was 'no such thing as climate change', and because we were mindful of not skewing the distribution, these participants were also coded as 4 .

Political ideology. Political ideology was measured by asking participants to rate their political values on two scales: how liberal or conservative they are $(1=$ very liberal, $9=$ very conservative) and how left or right they are ( $1=$ left, $9=$ right).

Demographics. Age was recorded (in years) using an open-response format. Gender was recorded by asking people if they were male, female, or 'other'. The 12 respondents who nominated the 'other' category were excluded from analyses in which gender was included. To measure education, we asked participants 'What is the highest level of education you have completed?' $(1=$ less than high school, $2=$ high school, $3=$ trade qualification, $4=$ university degree, $5=$ postgraduate degree).

Ethics statement. This research was approved by the Behavioral and Social Sciences Ethical Review Committee of the University of Queensland (ethics clearance no. 2015000181). In advance of completing the survey, respondents were provided with an information sheet describing the type of questions they would be asked to complete. The information sheet also emphasized the anonymity of data and their right to withdraw from the study. Choosing to complete the survey beyond that point was interpreted as informed consent. 
Note that SSI panelists agree to join the SSI panel through an opt-in registration process. By becoming a panel member, they are agreeing to participate in survey research. Individuals engaged by SSI through third-party sources demonstrate their consent to participate in survey research by entering the SSI system and completing a survey.

Data availability. The data that support the plots within this paper and other findings of this study are available at https:/osf.io/qzxv9/?view_only= 852910a2c08c42018edf84a0b556aa14.

\section{References}

40. Arnold, D. Why Princess Diana conspiracies refuse to die. The Conversation (30 August 2017); http://theconversation.com/whyprincess-dianaconspiracies-refuse-to-die- 82363

41. Hornsey, M. J., Harris, E. A., \& Fielding, K. S. The psychological roots of anti-vaccination attitudes: A 24-nation investigation. Health Psychol. 37, 307-315 (2018).

42. Kahan, D. M. in Handbook of Risk Theory (eds S. Roeser, S. et al.) 725-759 (Springer, Dordrecht, 2012). 